\title{
Les artistes urbains belges, la « crise migratoire » et le terrorisme. Entretiens avec Kaer, Gioia Kayaga, Giacomo Lariccia, David Mendez Yepez, Tutu Puoane et Rival
}

Belgians Urban Artists, the "Migration Crisis" and Terrorism. Interviews with Kaer, Gioia Kayaga, Giacomo Lariccia, David Mendez Yepez, Tutu Puoane and Rival

Belgas artistas urbanos, la «crisis de la migración» y el terrorismo. Entrevistas con Kaer, Gioia Kayaga, Giacomo Lariccia, David Mendez Yepez, Tutu Puoane y Rival

\section{Marco Martiniello}

\section{OpenEdition}

Journals

Édition électronique

URL : https://journals.openedition.org/remi/8226

DOI : 10.4000/remi.8226

ISSN : $1777-5418$

Éditeur

Université de Poitiers

Édition imprimée

Date de publication : 1 décembre 2016

Pagination : 147-161

ISBN : 979-10-90426-29-0

ISSN : 0765-0752

\section{Référence électronique}

Marco Martiniello, « Les artistes urbains belges, la « crise migratoire » et le terrorisme. Entretiens avec Kaer, Gioia Kayaga, Giacomo Lariccia, David Mendez Yepez, Tutu Puoane et Rival », Revue européenne des migrations internationales [En ligne], vol. 32 - $n^{\circ} 3$ et 4 | 2016, mis en ligne le 01 décembre 2018, consulté le 15 avril 2022. URL : http://journals.openedition.org/remi/8226 ; DOI : https://doi.org/ 10.4000/remi.8226 


\section{Les artistes urbains belges,} la " crise migratoire " et le terrorisme. Entretiens avec Kaer, Gioia Kayaga, Giacomo Lariccia, David Mendez Yepez, Tutu Puoane et Rival

\section{Marco Martiniello ${ }^{1}$}

Comme la France et bien d'autres pays occidentaux et non occidentaux, la Belgique est un pays d'immigration de très longue date. Les historiens n'hésitent pas à dire que ce bout de territoire, appelé Belgique depuis 1830, a en réalité toujours été une terre de brassage de populations depuis la préhistoire (Morelli, 2004). Le pays a ainsi célébré, durant toute I'année 2016, le soixantedixième anniversaire des accords bilatéraux entre les gouvernements belge et italien qui ont présidé à l'arrivée des mineurs italiens dans les charbonnages belges. L'immigration vers la Belgique n'a pas cessé depuis 1946, même si elle s'est diversifiée, tant au niveau des origines nationales des migrants, que des types de migrations (Martiniello et Rea, 2012). Les villes belges se caractérisent par une diversité ethnique, culturelle, religieuse et socio-économique croissante qui les enrichit, mais qui au même moment, défie les modes traditionnels de gestion publique dans tous les domaines (éducation, santé, emploi, arts et culture, politique, etc.). Dans la région bruxelloise, par exemple, près de $60 \%$ de la population est $d$ 'origine étrangère et pratiquement toutes les nationalités de la planète sont représentées (Hermia et Vandermotten, 2015).

Comme la France et bien d'autres pays occidentaux et non occidentaux, la Belgique fait face depuis plusieurs années à une arrivée importante de demandeurs d'asile en provenance de zones en guerre ou en instabilité politique endémique. Certes, il n'y a pas de "jungle de Calais " dans le pays, mais en 2015, le parc Maximilien s'était transformé en un camp ouvert en plein centreville pendant plusieurs semaines, dans lequel volontaires et demandeurs $\mathrm{d}^{\prime}$ asile tentaient à leur manière de répondre concrètement à la soi-disant " crise migratoire ". L'arrivée, l'accueil et l'intégration de ces personnes déplacées questionnent la société marquée par une polarisation forte au sujet des réalités migratoires actuelles. D'un côté, l'élan de solidarité est manifeste. De l'autre, les attitudes de replis et de rejet des demandeurs d'asile le sont tout autant.

1 Sociologue, Directeur de recherche au FRS-FNRS, Université de Liège, FASS-CEDEM, Bâtiment 31, boîte 24, Quartier Agora, Place des orateurs, 3; 4000 Liège (Sart-Tilman), Belgique;m.martiniello@ulg.ac.be 
Comme la France et bien d'autres pays occidentaux et non occidentaux, la Belgique a été récemment fortement marquée par des actes terroristes meurtriers d'une ampleur historiquement inconnue. Perpétrés par des personnes le plus souvent nées et socialisées dans les villes belges, ces actes terroristes, et les réponses sécuritaires qui y ont été données, ont un impact direct sur la vie des citoyens et des résidents et notamment sur la cohabitation au quotidien dans I'espace public urbain.

Dans ce contexte difficile, il semble opportun de recueillir, à l'occasion de cet anniversaire de la revue, la voix d'acteurs-clés des dynamiques urbaines belges sur ces questions, à savoir les artistes et en particulier les artistes dont l'histoire personnelle est intimement liée à la migration. La sociologie de l'immigration a trop souvent négligé les pratiques artistiques des immigrés et de leurs descendants (Martiniello, 2015). Pourtant, ces pratiques, autant que les artistes ayant une expérience migratoire, peuvent jouer un rôle central dans les processus de mobilisation sociale et politique de nos sociétés multiculturelles et souvent apporter une certaine fraîcheur dans des débats trop stéréotypés. Cet article est consacré à des chanteu(se)rs, musicien(ne)s, rappeu(se)rs et slameu(se)rs basés à Anvers, Liège et Bruxelles. Les six artistes qui ont accepté très aimablement de répondre à mes questions viennent donc de Flandre, de Wallonie ou de Bruxelles. Ils évoluent dans des univers artistiques différents du rap, au jazz, au slam en passant par la pop latina ou encore la chanson italienne. Ils ont chacun(e) une histoire migratoire particulière. Mais toutes et tous jettent un regard attentif et souvent inquiet sur l'évolution de nos sociétés multiculturelles dont ils sont des représentants incarnés, qu'ils le veuillent ou non. Les entretiens ont été réalisés en binômes durant l'été 2016.

Kaer, de son vrai nom Didier Gosset, est né à Liège d'une mère équatorienne et d'un père belge et musicien. II a grandi à Liège en Belgique, en Équateur et en Colombie. Pour Kaer, l'immigration « ça fait vraiment partie de son histoire, de ses racines ", même s'il se sent belge avant tout. À l'adolescence, il découvre le hip-hop d'abord en tant que grapheur, puis en tant que rappeur. II deviendra un des MC du légendaire groupe de rap Starflam. II mène en parallèle une carrière solo et toute une série d'activités à caractère social et culturel avec des publics fragiles comme les jeunes et les détenus. Son album Versatil, sorti en 2012 et enregistré en Équateur, fait la part belle aux rythmes latino et au rap en espagnol, marques de la biculturalité assumée de l'artiste liégeois (https://www. youtube.com/watch?v=gj_V35I1hBk).

Gioia Kayaga, de son vrai nom Gioia Frolli, est née en Belgique d'une mère belgo-burundaise et d'un père belgo-italien. "Donc, moi, je suis à moitié belge, un quart italienne, un quart burundaise. Je suis un pur produit de l'immigration ", dit-elle. Fascinée par la littérature en général et par le roman et la poésie en particulier, elle étudie les langues romanes aux universités de Namur puis de Bruxelles où elle s'installe. Elle y découvre le monde du slam. Après des expériences artistiques à Madagascar, à la Réunion et au Québec, elle monte en 2016 son premier spectacle complet, Tram 25, qui inclut notamment son superbe texte sans concession sur la Belgique d'aujourd'hui, Mon Pays (https:// www.youtube.com/watch? $v=t I I S h H K m D g Y)$. En parallèle à sa carrière artistique, Gioia est aussi professeur en alphabétisation pour des adultes pour la plupart étrangers. Elle travaille donc avec des " nouveaux migrants" au quotidien. 


\section{Photo 1 : Kaer}

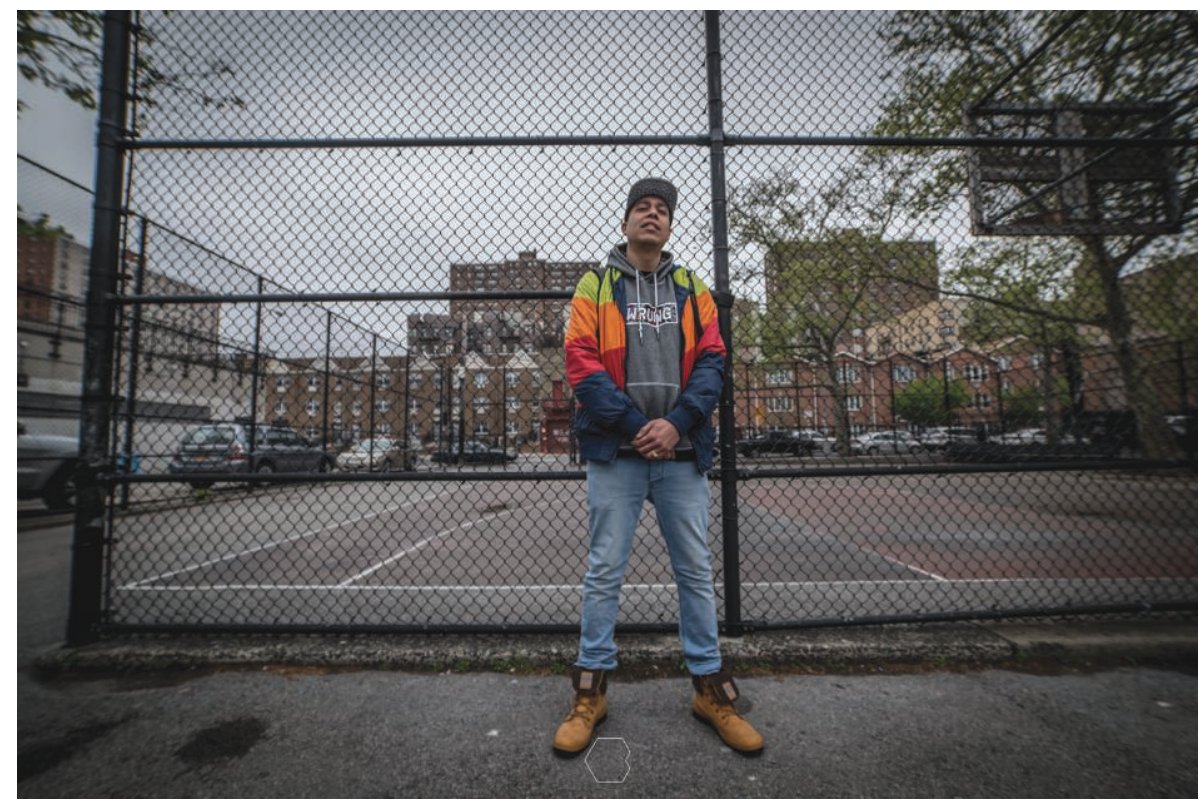

Crédit : Soke La Cabane.

Photo 2 : Gioia Kayaga

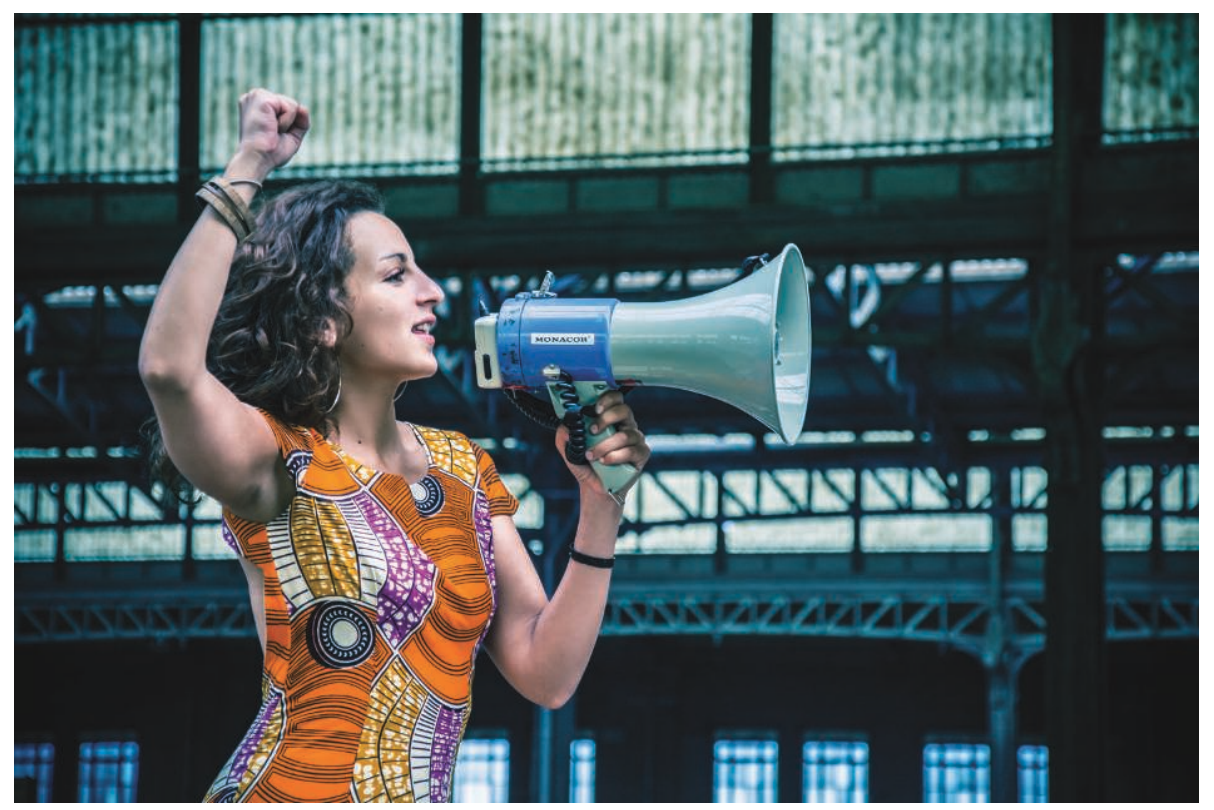

Crédit: GV photographe. 
Giacomo Lariccia est né à Rome. Après ses études universitaires, il arrive en Belgique en 2000 pour passer quelques mois au Conservatoire de musique de la ville de Bruxelles. "Après le conservatoire, je suis resté parce que j'ai trouvé toute une série de conditions qui $\mathrm{m}^{\prime}$ ont permis de continuer à faire de la musique, qui m'ont permis de faire une famille, d'avoir des enfants. C'était tout un mélange de conditions qui m'ont convaincu que rester en Belgique était une belle chose ", dit Giacomo. Guitariste de jazz de formation, il découvre le pouvoir des mots et devient auteur-compositeur-interprète. II gagne un important prix en Italie, le Premio Tenco. Son récent album, intitulé Sempre avanti [Toujours en avant], raconte l'épopée des mineurs italiens en Belgique après la Seconde Guerre mondiale. Giacomo se positionne ainsi comme le lien entre la vieille et la nouvelle immigration italienne en Belgique (https://www.youtube.com/ watch?v=QL67_XkhL5c\&list=PLrTHBPaLmL687d7Nz-xwU_eSuK1LeUNuD).

\section{Photo 3 : Giacomo Lariccia}

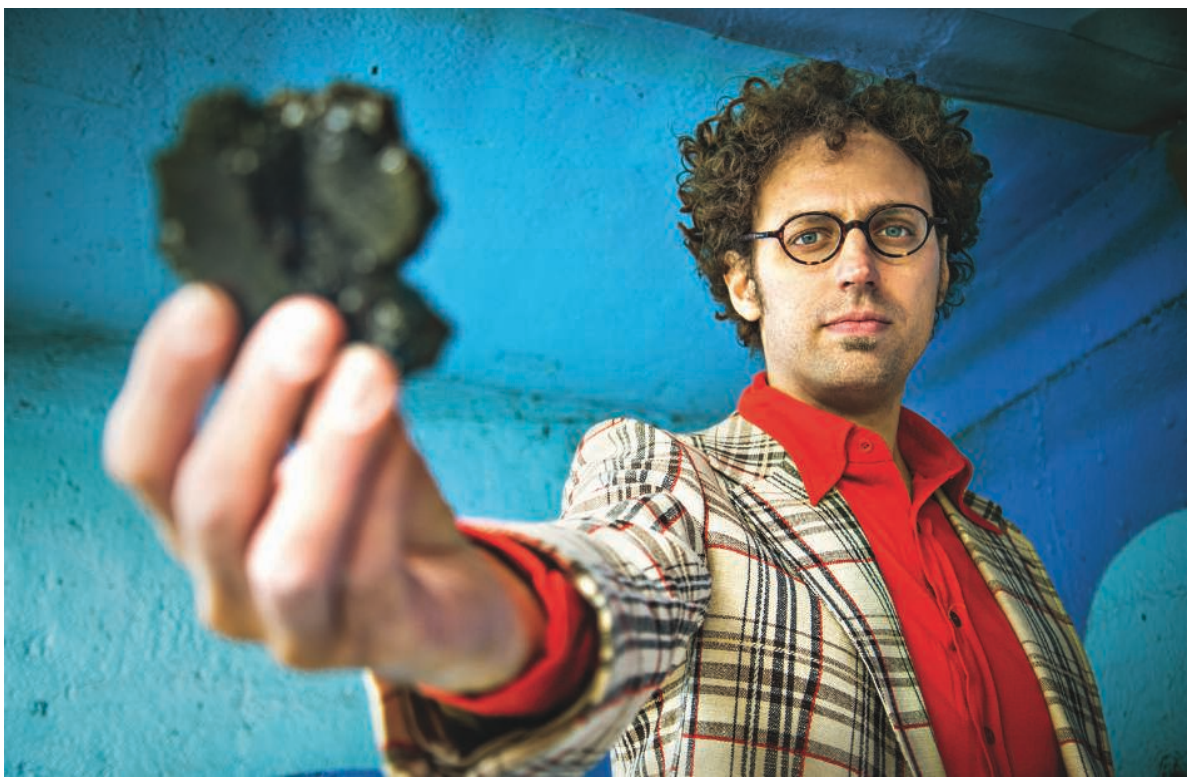

Crédit : Alessandro Vecchi.

David Mendez Yepez est né en Belgique. II vit à Bruxelles. Ses parents sont venus du Pérou faire une thèse de doctorat en Belgique. Leur rêve était de rentrer au pays pour contribuer à sa transformation. Hélas, la situation politique au pays a rendu ce rêve impossible à concrétiser. La famille s'est définitivement installée en Belgique. "Et du coup, mon rapport avec l'immigration est double. D'abord, je suis péruvien. Ma langue maternelle, c'est l'espagnol. Mes parents m'ont éduqué de manière sensiblement sud-américaine. Mais c'est aussi le fait $d^{\prime}$ avoir vu dans les yeux d'une mère et d'un père la difficulté que c'est de ne pas pouvoir retourner dans leur pays qu'ils aimaient beaucoup et qu'ils aiment encore. Et surtout, de ne pas pouvoir le transformer comme ils voulaient le faire. Et donc c'est un peu un rapport à la fois de migrant même, de jeune migrant, mais aussi un regard sur les migrants, donc sur mes parents ", dit 
David. II réussit brillamment ses études universitaires. II devient le leader de la plus grande association d'étudiants de Belgique francophone, mais la musique l'obsède. II décide de tenter la carrière musicale avec son groupe Chicos y Mendez qui cultive ce que David appelle "I'alegría militante " (https://www. youtube.com/watch?v=r52u_9IM-TE).

Photo 4 : Chicos y Mendez

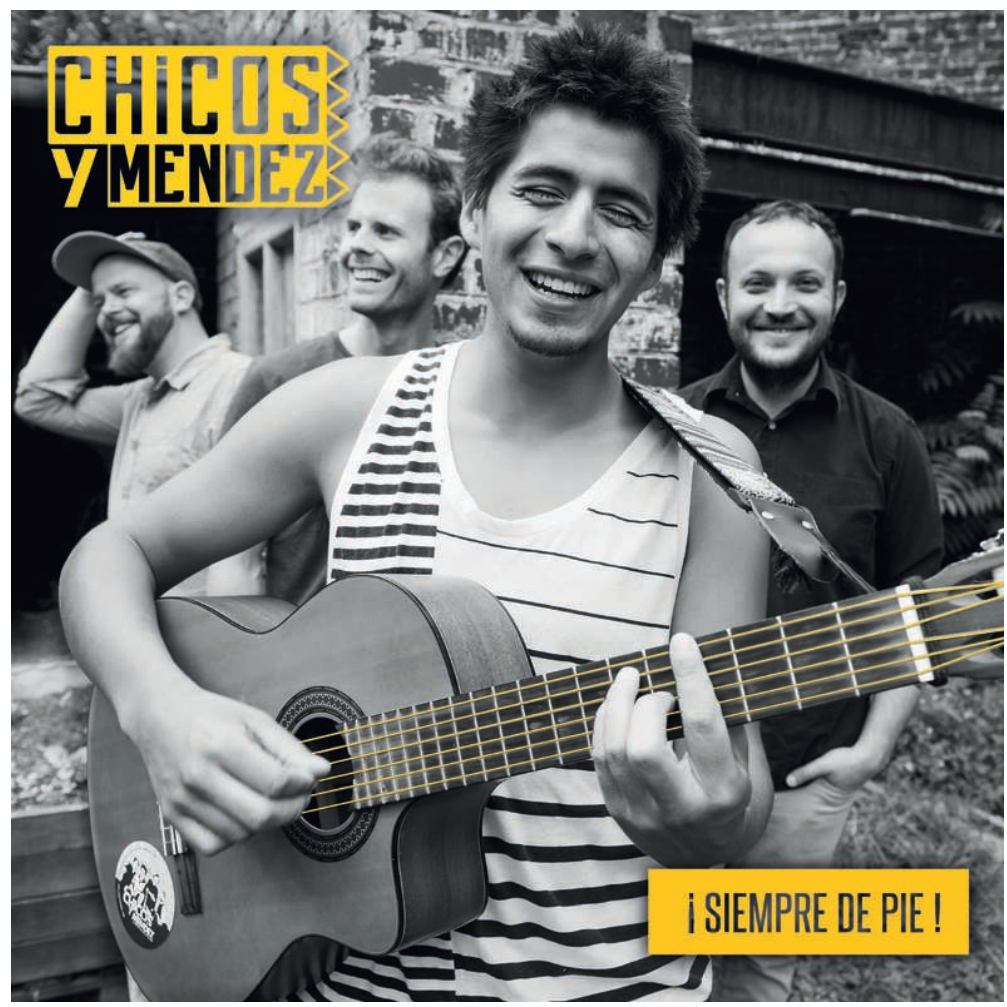

Crédit : GrooveMan.

Tutu Puoane est née dans le township de Atteridgeville en Afrique du Sud dans la région de Pretoria. Elle a quinze ans lorsque s'effondre enfin l'abject régime de l'apartheid. C'est paradoxalement après la chute de ce système qu'elle se rend réellement compte de son impact sur sa vie. À cette époque, elle passe d'une école totalement " noire " à une école " mixte " dans la ville de Johannesburg. Elle y rencontre pour la première fois des adolescents blancs et le racisme explicite. " J'ai toujours été la chanteuse. Je ne voulais rien d'autre. $C^{\prime}$ est vraiment mon identité depuis la maternelle ", dit Tutu Puoane. Elle étudie dans une académie d'arts et de musique réservée aux noirs puis au collège de musique à I'Université du Cap. Elle obtient une bourse pour poursuivre sa formation à La Haye aux Pays-Bas. Elle poursuit sa route vers Anvers I'année suivante. Elle $s^{\prime} y$ installe et gagne progressivement une excellente réputation sur la scène jazz de la ville et du pays. Son projet le plus récent rend hommage à Joni Mitchell (https://www.youtube.com/watch?v=p-thl3h7VKk). 
Photo 5 : Tutu Puoane

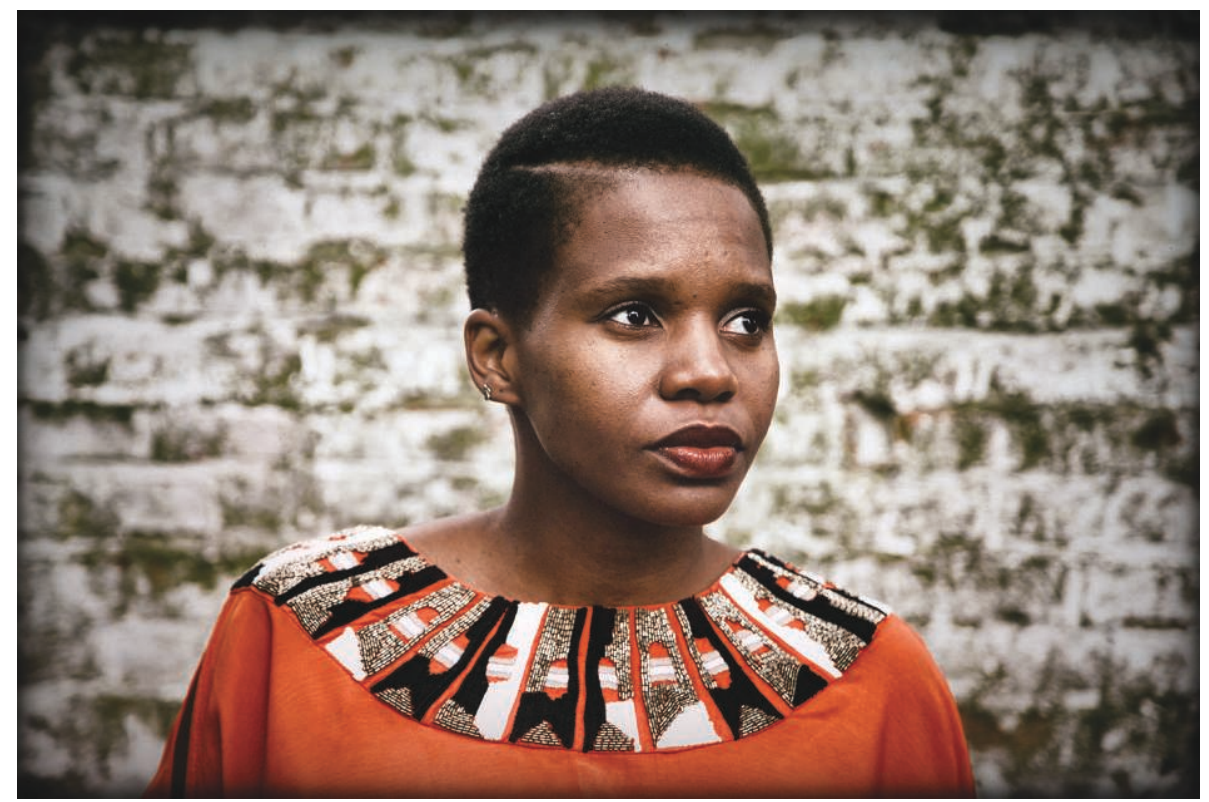

Crédit : Hugo Van Beveren.

Rival, alias Youssef, est né dans la région bruxelloise dans une famille immigrée originaire du Maroc. Son père, auparavant boucher-abatteur à Casablanca, est arrivé en Belgique dans le cadre des accords bilatéraux d'immigration entre la Belgique et le Maroc vers la fin des années 1960. Sa mère a élevé les cinq enfants de la famille. Très tôt touché par le virus du hip-hop, Rival est depuis près de vingt-cinq ans une des figures centrales du hip-hop belge. II fonde le crew CNN (Criminels Non Négligeables) en 1991. Son album, De la rue à la scène, sorti en 1999, reste un classique du rap belge. Il est membre et fondateur de la structure Souterrain Productions à qui on doit la première compilation hip-hop Belge $100 \%$ indépendante et de nombreux autres évènements majeurs qui ont structuré le hip-hop belge. Membre de la Zulu Nation, Rival est aussi un proche de beaucoup de grands noms du hip-hop international. Son projet récent, NOUéVOU, I'unit à une violoniste-chanteuse juive et à un beatboxeur d'origine turque. Ensemble, ces artistes font exploser les frontières entre les disciplines artistiques (rap, klezmer, beatbox) et ils dépassent allègrement les barrières ethniques et religieuses (https://www.youtube.com/watch?v=VmwZ1GqaQU0).

Trois questions ont été abordées avec ces six artistes aux profils très diversifiés : la " crise migratoire " actuelle, l'impact des attaques terroristes qui ont secoué le pays et des politiques mises en œuvre pour y répondre et enfin, la manière dont ils conçoivent leur rôle en tant qu'artistes dans la période très délicate que nos sociétés traversent. 
Photo 6 : Rival Cnn

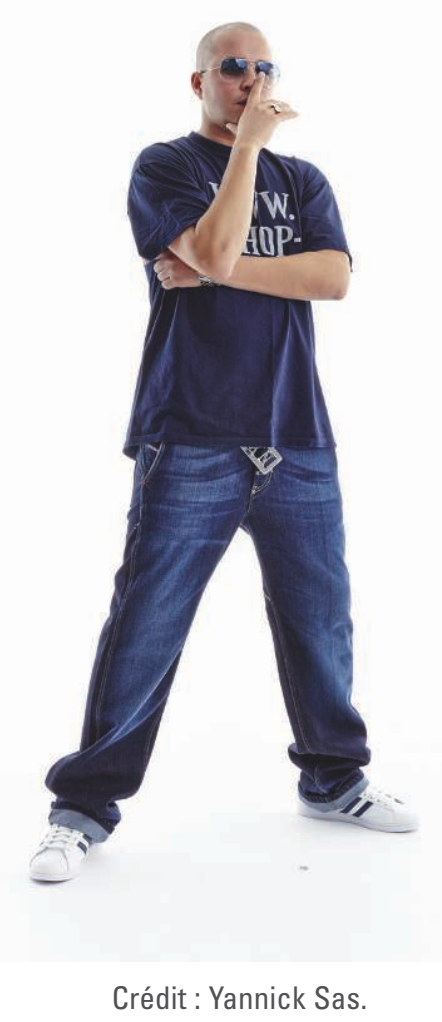

\section{Les artistes face à la u crise migratoire "}

Le nombre de candidats à l'exil qui périssent en Méditerranée lors de la traversée ne cesse de croître. Les réponses politiques données par les gouvernements européens et par l'Union européenne ne semblent pas être à la hauteur des enjeux révélés par ces déplacements de population. L'opinion publique en Europe semble se polariser. D'un côté, l'élan de solidarité envers les réfugiés est manifeste. De l'autre, les pratiques du rejet de ces nouveaux arrivants semblent se banaliser. Que pensent les artistes de cette situation hautement complexe?

Pour Kaer : " À l'ère de la mondialisation, la politique internationale met en place des conflits pour permettre l'exploitation des zones qui sont riches en productions agricoles, mais aussi en productions énergétiques. C'est un monde en guerre surtout au Moyen-Orient. $C^{\prime}$ est un monde en guerre où on a vécu 
beaucoup la question des réfugiés. II y a eu les boat people, les Vietnamiens, les Cambodgiens qui arrivaient. On les a accueillis. II y a eu les vagues d'immigration subsahariennes. Et c'était chaque fois des réfugiés. II y a eu les Chiliens. Puis il y a eu toute la vague aussi de I'ex-Yougoslavie, les Albanais, etc. J'ai l'impression qu'on vit un truc maintenant, mais ce n'est pas nouveau. II y a toujours eu ça. Le monde change. Et nous, on voudrait ne pas changer. Maintenant, on se rend compte qu'il y a une arrivée massive de gens qui viennent de ces zones de guerre. On a peur et je pense à juste titre. On se rend compte aussi que dans ces flux migratoires, il y a des gens qui sont là pour venir semer la terreur chez nous. On ne peut pas le nier. Ça reste une proportion infime par rapport au nombre de gens qui, vraiment, fuient cette guerre pour survivre, mettre leurs enfants à I'abri. Tenter leur chance, tout simplement. On ne peut pas laisser les gens mourir en Méditerranée sans fin. C'est un vrai cimetière la Méditerranée. Si on consacrait les moyens à vraiment investir dans l'accueil de ces personnes, I'intégration de ces personnes, au lieu d'acheter des F16 et d'aller bombarder la Syrie ? La haine attire la haine. C'est quand même dingue de se dire qu'on va bombarder des régions où déjà l'État islamique est implanté avec des populations civiles qui sont sous le joug de ces gens-là quoi. Ils sont des boucliers humains ! On est complètement dans une ère de répression. Mais on ne répond pas à un problème, en aggravant le problème. Voilà, je pense qu'il y a de la place pour tout le monde".

Gioia Kayaga souligne la peur et la méconnaissance de la population belge : " II y a vraiment un racisme qui se crée par cette méconnaissance et moi je sais que, en fréquentant ces personnes qui arrivent tous les jours, il y quelque chose de très fort qui peut se faire au niveau du rapport humain. Je pense que les médias ont un rôle parfois très important. J'ai l'impression qu'il y a un repli très fort sur soi-même avec la question, notamment, de l'islam qui est vraiment très fort dans cette question de la migration. La question de la religion ajoute encore un point de tension parce que c'est sûr que, culturellement, c'est des choses qui sont très éloignées de nous et donc on a du mal à les accepter, à les comprendre. Le voile, la pudeur, ce sont des choses que nous, on a totalement perdu. Le problème, c'est que ce n'est pas que migratoire comme crise, c'est économique, c'est à plein de niveaux de la société et donc les gens ont peur, je pense, avant tout, de perdre ce qu'ils ont, de perdre leur confort. Beaucoup de gens s'accrochent à leur petit confort et n'ont pas envie qu'on vienne. Ils ont l'impression vraiment de vivre une intrusion alors que ce n'est pas directement dans leur maison que les gens vont débarquer. Pour moi, il faut par tous les moyens, essayer d'échanger, de trouver des moyens de discuter, de communiquer. Et alors, on se rend compte que toutes les idées qu'on pouvait avoir sont parfois totalement fausses. Et même moi, je pouvais avoir des idées sur l'islam que j'ai vraiment relativisées en connaissant des musulmans. Je pense que c'est avant tout un problème d'aller vers l'autre ".

Giacomo Lariccia affirme que : "C'est une affaire très complexe qui concerne la guerre au Moyen-Orient, l'intégration en Europe de ces flux migratoires et aussi, bien sûr, le côté religieux. Je trouve que si, en Europe, on veut continuer à accueillir les personnes qui s'éloignent de leur pays parce qu'ils ont des difficultés comme la guerre, il faut que tout se fasse dans un contexte qui permet que les gens arrivent dans des conditions de sécurité. Deuxième chose, qu'ils puissent s'intégrer en Europe. Donc, pour moi, je pense que la solution peut 
être seulement l'octroi de visas aux personnes qui partent. Les obliger à faire ce voyage qui trop souvent se termine par des centaines de morts dans la Méditerranée est inacceptable ".

David Mendez Yepez : "Comme compositeur et chanteur, je trouve que les mots sont très importants. Le mot crise en fait, ça veut tout et rien dire. Quand on dit la crise des migrants, je pense qu'avant toute chose, il faut voir ça comme une crise de l'accueil et il faut l'appeler comme ça. Parce que si on appelle ça une crise des migrants, on met la faute sur les migrants alors qu'eux, ils fuient leur pays, ils sont les premières victimes de cette situation. J'ai l'impression qu'on est arrivé à un moment où les inégalités sont tellement exacerbées ; le monde est dans une situation terrible. L'immigration, c'est un peu une double punition. Ils doivent quitter leur pays et en plus, on les considère comme des poids pour la société. Donc, là, on crée déjà un terreau qui est très fertile pour toutes sortes d'exclusions".

Tutu Puoane est très affectée par cette " crise migratoire " : "Ces gens sont moi. Ils sont moi. Je suis juste chanceuse d'être née dans une autre partie de I'Afrique. J'aurais pu facilement être née ailleurs en Afrique et devoir moi aussi nager jusqu'ici. C'est très déprimant ".

Enfin, Rival rejoint Tutu : "Quand je regarde les vidéos, je les enchaîne. Tu pleures parce que tu te dis "voilà ce que certains subissent, voilà ce que d'autres ne veulent pas dire". Tu te rends compte à quel point on est dans une espèce d'impuissance... ce train de l'enfer est en train de passer à grande vitesse et tu as l'impression qu'il a un chemin à parcourir et que rien ne va l'arrêter et qu'il est sur ses rails fermement dessus et qu'il trace. Tu vois et toi, tu es là et tu regardes et tu te dis "ça se dégrade". Comme si tout cela faisait partie d'une équation qui nous dépasse. On multiplie les actions. Ils nous divisent pour mieux régner. On additionne espoir, amour, humanité, art, cultures et le triste sentiment que le futur lui-même s'y soustrait. Alors j'écris un rap comme pour me venger. J'organise des évènements pour tromper le sort, mais quoi que je fasse, je le fais par conviction simple : tant qu'à être un passant, je ne serai pas invisible et sans voix ".

Tiraillés entre les tentatives d'analyse, la colère, l'émotion et l'empathie, ces artistes comme tous les citoyens sont clairement affectés par cette " crise migratoire " qui révèle des enjeux géopolitiques, économiques et culturels globaux qui dépassent largement la question des migrations. Mais entre-temps, les candidats à l'exil continuent jour après jour à donner leur vie à la mer dans l'espoir de la vivre sur terre.

\section{Les artistes face au terrorisme}

Plus de cinq mois après les attentats de Bruxelles, la Belgique est toujours en état d'alerte. Le niveau officiel de la menace est de trois sur une échelle de quatre. Cela se concrétise notamment par une présence renforcée des forces armées et de la police dans les espaces publics et par des contrôles lors de toutes les manifestations et tous les événements publics. Les artistes au cœur de cet article sont profondément urbains. Leur terrain d'action, c'est la ville et ses quartiers. Comment analysent-ils cette donne du terrorisme qui force la plupart d'entre nous à changer en partie notre mode de vie au quotidien ? 
Pour Kaer : «Il y a non seulement un clivage qui s'installe du côté de ceux qui se radicalisent et qui essaient de dire "oui, mais c'est une guerre contre l'islam. Vous ne nous avez jamais acceptés parce qu'on est d'une religion différente". Mais il y a un radicalisme de l'autre côté aussi. Des locaux qui disent "vous ne vous êtes jamais intégrés à notre culture. Vous êtes différents de nous, vous ne nous aimez pas". Donc, on est dans un radicalisme de part et d'autre. Moi, les discours hippies "Oui... on est tous pareils, on va jouer du djembé, faire un barbecue hallal et on va manger ensemble", tout ça... Moi, je crois que ce n'est pas ça. Je crois qu'on devrait vraiment mettre en place l'Affirmative Action, c'est ça le truc, la discrimination positive. Je trouve que le nom en français est horrible parce que la discrimination positive, c'est vraiment du pipeau au niveau du nom. Mais je crois à ça. On devrait travailler ensemble. Je pense qu'en travaillant ensemble, en résolvant des problèmes ensemble qui sont liés à une tâche commune, un objectif commun, on apprend à se connaître ".

Gioia Kayaga s'étonne que : «Beaucoup de gens aiment bien le fait qu'il y ait des militaires partout. Et moi, c'est totalement le contraire. C'est quand je vois les militaires que je me sens en insécurité. Sinon, le reste du temps, je suis dans ma ville. Les choses concrètement $n^{\prime}$ ont pas changé. Et, $c^{\prime}$ est chaque fois que je vois les militaires que ça me rappelle que, voilà, il y a des choses tragiques qui ont eu lieu. La réponse au terrorisme a été extrêmement sécuritaire. En France, c'est la même chose. C'est tellement facile. On reporte le problème sur l'islam au lieu de se poser des questions de société, de se dire que, actuellement, dans notre société en Belgique, il y a des jeunes, qu'ils soient nés en Belgique ou pas, qui préfèrent mourir que vivre. Et pour moi, souvent, on oublie que c'est des jeunes qui sont prêts à se suicider, d'abord. C'est des jeunes qui sont prêts à mourir. Et puis, après, évidemment, ils tuent d'autres personnes pour donner aussi un coup d'éclat à leur mort. Je pense qu'on a souvent tendance à occulter qu'il y a un vrai désespoir chez les jeunes dans notre société du fait que, les perspectives d'avenir sont parfois, en fonction du milieu d'où on vient, extrêmement réduites. Et voilà, moi, je fais les ateliers de slam dans les écoles avec les jeunes et tout ça donc je le vois. II y a beaucoup de jeunes, ils disent “ben, moi j'sais que je vais être au chômage. Là, je suis en filière éducation, machin". Quelle perspective d'avenir on a à leur offrir ? C'est plutôt les gens qui ont de réels problèmes et qui se reportent sur l'islam. Ils utilisent l'islam comme manière de se valoriser, souvent. II y a un problème de sentiment d'infériorité. On a l'impression qu'on n'est rien du tout et tout à coup, avec l'islam, on devient quelqu'un d'important. Pour moi, entre quelqu'un qui va descendre dans la rue et tirer sur les gens sans être musulman et un musulman qui va faire la même chose, il n'y a pas de différence dans l'acte. C'est un acte de désespoir, c'est un acte de révolte, de colère, de dégoût ".

Giacomo Lariccia partage en partie cette analyse. II constate que : "Très souvent, ces attaques sont faites aujourd'hui et même en France, par des Français, par des Belges. Il faut mettre en cause l'intégration : qu'est-ce qu'il y a eu qui a fait que ces personnes rejettent totalement toutes les valeurs européennes. Pourquoi ont-ils rejeté totalement les valeurs européennes ? De notre part, quelle intégration a-t-on pu offrir à ces personnes ? Et elles, acceptent-elles d'être intégrées dans notre société ?". 
Pour David Mendez Yepez : "La question qu'on doit se poser, ce n'est pas tellement comment le terrorisme voit le jour, mais plutôt comment est-ce que des jeunes de chez nous, qui ont grandi ici, ont pu être séduits par une idéologie de ce type-là. Et c'est ça la question. C'est le fait que notre identité européenne est assez monolithique. Elle n'a pas beaucoup évolué avec le changement de sa population. Un très bon exemple, c'est la France. On a 5 millions de musulmans en France. Pourtant, la République, elle est assez conservatrice, blanche, plutôt patriarcale. Du coup, l'image qu'on renvoie de ce qu'est le citoyen est complètement déphasée par rapport à la réalité. Et donc, qu'est-ce qui se passe sur le terrain ? Ben, effectivement, quand on a l'impression qu'on n'appartient à rien, c'est un déracinement total. Je pense que si la République, dans le cas de la France, ou le Royaume, dans le cas de la Belgique, avait changé de visage et avait assumé son côté multiculturel et qu'on en aurait fait une fierté..., François Hollande pourrait dire : "Si 5 millions de mes concitoyens sont musulmans, je suis un peu musulman également" ". Pour David Mendez Yepez, l'exclusion des jeunes Français dont les parents ou les grands-parents étaient des immigrés du roman national sur la base de leur appartenance religieuse doit être prise en compte pour rendre compte du phénomène d'aliénation qui peut conduire au choix djihadiste.

Alors que Tutu Puoane s'exprime peu sur la question du terrorisme si ce n'est pour réitérer le choc qu'a été pour elle personnellement l'attentat à l'aéroport de Bruxelles, elle qui était encore dans la place vingt-quatre heures avant l'explosion, Rival est plus loquace : "Tu ressens un grand mal-être parce que les gens sont paumés et aujourd'hui les inégalités sont beaucoup plus visibles. Tu as le net et donc tu as de l'information, de la contre-information et le net est un outil à double tranchant. Certaines inégalités sont relativement dangereuses quant à la valeur de la vie humaine qui est à géométrie variable. La vie humaine a un prix qui varie selon les intérêts financiers et la force de certains lobbies. Quand tu vois qu'on s'insurge par rapport à certains pays alors que des drames frappent dans d'autres et on ne dit rien. Le Congo, ce qui se passe en Birmanie et j'en passe. La Turquie, y a eu des attentats. À l'Euro de football, si je ne me trompe pas, ils n'ont fait à aucun moment un hommage aux victimes. Et ça m'a fait mal parce que tu te dis "mais pourquoi on s'obstine à être aussi maladroits et aussi inhumains". Parce que ce qui se passe n'est pas juste, n'est pas acceptable, il n'est pas équitable et c'est ce qui nourrit, malheureusement d'autres qui se cachent et justifient les actes (terroristes) par "voilà, nous ne comptons pas pour eux. Ils choisissent ceux qui pour leurs critères sont légitimes en tant que victimes. Nous ne sommes pas des victimes dignes, nous ne sommes pas des vies humaines dignes d'être reprises dans la presse". Quand tu regardes, tu te dis "mais c'est tellement facile de pousser quelqu'un au crime en brandissant l'injustice et l'inégalité mondiale, tu vois ?"Y a des choses où tu regardes et tu te dis "qu'est-ce qu'on va faire... ? Qu'est-ce qu'on va faire ... ?" ".

Au-delà des différences de compréhension et d'interprétation de la situation actuelle marquée par le terrorisme, les artistes soulignent tous à leur manière la nécessité de s'efforcer de comprendre et d'expliquer, certes non pas pour excuser les actes terroristes, mais pour tâcher d'imaginer des moyens de mettre un terme aux violences aveugles partout dans le monde, et pas seulement dans nos villes européennes. 


\section{Que peuvent faire les artistes ?}

Que peuvent faire les artistes face à l'immensité des défis d'aujourd'hui ? $\mathrm{C}^{\prime}$ est le dernier thème de discussion abordé avec eux dans cet article.

Kaer a une opinion claire : "Je ne suis pas un travailleur social, je ne suis pas un homme politique. Moi, j'ai envie de faire de la musique, c'est vrai, qui retranscrit ce que je vis et qui peut retranscrire aussi ce qu'on vit. Quand on se positionne en tant qu'artiste, on essaie de parler de son monde, de son regard sur le monde. Et évidemment, on peut être dans le texte sérieux, on peut être dans la réflexion, dans l'utopie quelque part. Construire une utopie, la chanter, la vivre et essayer que les gens se retrouvent autour de cette idée. Mais on peut être aussi dans le lâcher-prise. On peut être aussi dans I'humour, la haine, la joie. Moi, c'est comme ça que je conçois maintenant mon rôle. Avec Starflam, on nous a collé une étiquette. Des groupes comme NTM, comme IAM, on les a pris pour les haut-parleurs de la banlieue, les délégués syndicaux des jeunes de banlieue. Je pense que moi, en tant qu'artiste, mon rôle n'est pas de trouver des solutions, de proposer des solutions. Peut-être dans l'une ou l'autre chanson, mais je n'ai pas envie d'y dédier ma vie. Je n'ai vraiment pas envie de ça. J'ai changé, c'est clair. Quand j'ai commencé Starflam, on était dans une époque où, nous, on sortait de l'adolescence avec tout ce que ça implique comme valeurs, envie de rébellion, utopie, construisons un monde nouveau ensemble, tu vois? Toutes ces belles choses. Et une époque où on était dans de grosses manifs étudiantes. Tous les jeunes descendaient dans la rue, contre les réformes. On s'est fait tabasser par les flics dans les tunnels ici à Liège. On était dans cette lutte où on se réunissait. C'était une espèce de mini Mai 68 qui n'a pas abouti sur un énorme changement, mais qui a rassemblé les gens. Par notre discours, on rassemblait les gens autour de cette énergie-là. Donc, on a conservé cette étiquette de groupe adolescent revanchard, tu vois ? Et qui ne disait pas des trucs cons. On nous a reconnus intellectuellement. On était quand même dans la recherche que ce soit du champ lexical, du vocabulaire de l'idée et aussi, de la référence. Est-ce qu'à quarante ans passés, on est toujours là-dedans ? Est-ce que ça ne m'a pas renfermé dans un truc qui, artistiquement, peut me bloquer parfois ? Je n'ai pas envie de passer mon temps à faire le rap de monsieur mondialisation qui se positionne et tout. Je fais beaucoup d'ateliers avec des jeunes. Et je vois les jeunes, ils s'amusent quoi. Ils font des trucs. Bon, je corrige des fois sur le fond un peu, j'essaie de remettre un peu l'église au milieu du village. Mais, je ne peux pas radicalement les changer dans leur optique, dans ce qu'ils écoutent et tout. Et je me dis, mais en fait ça sert à ça le rap. Ça sert à se sentir bien, à partager une énergie, un sentiment de liberté de quelqu'un qui, à un moment donné, n'a pas peur d'écrire, n'a pas peur de monter sur une scène. Est-ce que ce n'est pas ça aussi dont on a besoin ? La liberté ".

Gioia Kayaga n'est pas de la même opinion que Kaer : "En allant dans les soirées slam, je me suis dit, pour moi, c'est ça l'exercice de la démocratie. C'est que, chacun a un temps délimité, vient devant le micro et vient parler de ce qu'il veut. II y a une réelle qualité d'écoute qui $\mathrm{m}^{\prime} \mathrm{a}$ impressionnée les premières fois. Et, celui qui monte sur scène, il peut pousser un coup de gueule, il peut parler d'amour, il peut parler de ce qu'il veut, mais on a toujours cette obligation d'essayer de prendre le public avec soi. D'essayer, même pas forcément qu'il soit d'accord, mais qu'il nous écoute et peut-être qu'il comprenne. Et donc, 
moi, ça m'a vraiment fait travailler sur mon écriture et me disant, "voilà, moi je pense ça ou j'ai cette colère en moi ou cette histoire à raconter. Maintenant, il faut que je le formule et que je trouve le moyen que les gens, même s'ils sont pas d'accord avec moi, puissent, d'une manière ou d'une autre, essayer de comprendre ce que j'ressens". Et donc, ça m'a vraiment fait travailler sur mes textes. Je me suis dit voilà, chacun a la parole, chacun peut dire ce qu'il veut et petit à petit, on essaie de trouver les meilleures manières d'apporter ce qu'on veut dire. Et je trouvais ça vraiment intéressant. Et puis sinon, je ne me pose pas vraiment la question de "est-ce que ça va changer quelque chose ?". C'est plus quand j'ai quelque chose qui m'énerve ou qui me met en colère ou qui me révolte, j'ai besoin d'en parler, j'ai besoin de me vider via ce qui est mon outil, l'écriture et puis après, de mettre ça en musique. Mais c'est avant tout pour me libérer moi-même. Et après je me dis, ben, si je peux essayer que quelques personnes se reconnaissent dedans ou essayent de comprendre mon point de vue, c'est une bonne chose, mais je pense que c'est avant tout parce que c'est des sujets qui, moi-même, me touchent et qui me révoltent. Donc c'est chacun, ses préférences au niveau artistique, ce qui lui vient plus facilement. Comme je suis quelqu'un de très engagé et assez révolté, ça se retrouve naturellement dans mes textes. Mais je ne me dis pas que ça va changer quelque chose. Je ne me dis pas que voilà, c'est comme ça qu'on va forcément changer le monde, mais j'ai quand même l'impression qu'on prend la peine d'essayer d'organiser sa révolte, d'en faire un texte cohérent. Ça fait réfléchir. Et je sais que je suis quelqu'un qui peut être un peu trop cash, ou un peu trop extrême si je m'exprime directement. Et donc, je préfère avoir toujours cette prise de recul. $\mathrm{D}^{\prime}$ abord travailler mon texte et puis après de l'apporter et de ne pas m'exprimer à chaud sur tous les sujets qui viennent parce que c'est le meilleur moyen de dire des conneries. Écrire me permet la prise de recul ".

Quant à Giacomo Lariccia, il ne pense pas être capable de changer le monde, mais il dit: "Je pense que le seul fait de continuer à faire ce que je fais, chanter en italien, raconter des histoires, parfois des histoires sur l'immigration italienne, montre qu'un monde multiculturel est possible. Et déjà la réalité ! Chanter en italien en Belgique, c'est déjà un signe qu'on vit déjà dans un monde qui accepte les différences et qui doit apprendre à accepter d'autres différences encore. Donc, je peux continuer à faire ce que je fais parce que je pense que c'est un signe qui peut aider les gens aussi ".

David Mendez Yepez considère que : "La musique, c'est politique ". Mais, " il faut reconnaître une première chose, la musique, moi je l'ai faite parce que c'était une manière de vivre ma multiculturalité et de vivre en paix avec mon identité. Parce que c'est aussi une manière de revendiquer, chanter en espagnol, parler de l'Amérique latine, parler de la migration parce qu'il y a pas mal de chansons qui parlent de l'immigration, c'est aussi une manière d'exister. Et je ne sais pas si c'est sublimer ou exorciser, mais en tout cas, c'est peut-être essayer de transcender une douleur (collective, personnelle ou familiale) en quelque chose de lumineux. Après, quand je dis que la musique est politique, c'est plutôt parce qu'elle a vocation à être partagée. C'est un moment dans l'espace public. Quand on joue sur une scène ou quand on passe en radio ou quand on achète notre $C D$ ou quand il est sur Spotify ou autre, c'est un moment où on est dans l'espace public. Et ça, c'est sûr, c'est une manière de manifester dans I'espace public. Donc, c'est politique, pour moi. Le sens que, moi, je mets dans 
la musique aussi, c'est effectivement, d'une part, porter une parole dans l'espace public, traiter certains thèmes qui soient à la fois personnels ou quotidiens ou politiques. Et avoir, effectivement, l'ambition de traiter de certains sujets avec un autre angle $"$.

Rival rejoint d'une certaine manière David MendezYepez. II n'est pas gêné par l'étiquette d'artiste engagé. "Quand j'ai commencé ", dit Rival, " c'était pour moi tellement évident de faire du rap contre l'apartheid, la drogue, contre la violence. C'était ça le truc et j'ai toujours considéré que, voilà, tu fais de la musique, tu as la possibilité d'être entendu, tu as la possibilité d'être un orateur. Alors, respecte le temps et l'attention qui te sont accordés. J'essaye de faire au mieux. Dans mon titre Idéaliste, je dis : "[...] à celui qui comme moi écrit dans l'ombre et la solitude que tes mots soient lumière et que s'en fasse l'écho la multitude". J'ai toujours considéré qu'être engagé était ce qui différencie le rappeur du Master of Ceremony $(\mathrm{Mc})$ alors cette étiquette non, ça, ne me dérange pas. J'ai besoin au travers de tout ce que je fais d'essayer d'apporter mon intention de changement de nos quotidiens vers quelque chose de différent, de mieux, j'espère ".

Les ambitions de Tutu Puoane, sont beaucoup plus limitées. Elle s'inscrit plutôt lorsqu'elle en a l'occasion dans des actions humanitaires : " S'il y avait une chanson que je pourrais écrire qui pourrait tout résoudre, je le ferais. Mais des chansons comme ça ont été écrites tout au long de I'histoire et rien ne change. Donc, je ne pense pas que ce soit la seule responsabilité des artistes, de l'artiste en général. Je veux dire que tout le monde fait ce qu'il peut. Je fais ce que je peux, mais je ne suis pas un activiste. II y a d'autres artistes qui sont des militants. Cela dit, si on me demande de venir chanter pour le bénéfice des réfugiés, je le fais. Quand on me demande de chanter pour récolter de l'argent au profit des hôpitaux en Afrique, je le fais. Je fais ce que je peux. J'ai organisé moi-même, un concert pour récolter des fonds pour une fondation en Afrique qui donne des livres aux enfants. Donc, je fais vraiment ce que je peux ".

Nous faisons tous ce que nous pouvons face à une situation sur laquelle il semble très difficile d'avoir une prise directe. Les artistes, sont des acteurs clés, mais souvent négligés des débats actuels. Ils peuvent nous apporter du plaisir, nous faire réfléchir, parfois nous aider à nous mobiliser, toujours contribuer à rendre ce monde un peu moins insupportable. Dans le marasme actuel, c'est déjà çà.

\section{Références bibliographiques}

Hermia Jean-Pierre et Vandermotten Christian (2015) Le monde dans Bruxelles. Bruxelles dans le monde, Brussels Studies, 94, [en ligne]. URL : http://brussels. revues.org/1316

Martiniello Marco (2015) Immigrants, ethnicized minorities and the arts: A relatively neglected research area, Ethnic and Racial Studies, [online]. URL: http:// www.tandfonline.com/doi/full/10.1080/01419870.2015.998694

Martiniello Marco et Rea Andrea (2012) Une brève histoire de l'immigration en Belgique, Bruxelles, Fédération Wallonie-Bruxelles, 66 p.

Morelli Anne (2004) Histoire des étrangers et de l'immigration en Belgique, de la préhistoire à nos jours, Bruxelles, Couleur livres, 416 p. 


\section{Marco Martiniello}

\section{Les artistes urbains belges, la " crise migratoire " et le terrorisme. Entretiens avec Kaer, Gioia Kayaga, Giacomo Lariccia, David Mendez Yepez, Tutu Puoane et Rival}

Basé sur des entretiens menés avec six artistes de la scène musicale urbaine de trois grandes villes belges (Anvers, Bruxelles et Liège), I'article aborde trois questions : la " crise migratoire " actuelle, l'impact des attaques terroristes qui ont secoué le pays et les politiques mises en œuvre pour y répondre et enfin, la manière dont les artistes conçoivent leur rôle en tant qu'artistes dans la période très délicate que nos sociétés traversent. L'intérêt de ces conversations entre un sociologue des migrations et des artistes urbains dont l'histoire personnelle est directement marquée par les migrations repose sur l'idée que ces derniers sont des acteurs-clés dans les dynamiques urbaines post-migratoires et interculturelles.

\section{Belgians Urban Artists, the "Migration Crisis" and Terrorism. Interviews with Kaer, Gioia Kayaga, Giacomo Lariccia, David Mendez Yepez, Tutu Puoane and Rival}

Based on interviews with six artists from the urban music scene of three major Belgian cities (Antwerp, Brussels and Liège), the paper addresses three issues: the "migration crisis" today, the impact of the terrorist attacks that shook the country and the policies implemented to respond and finally, the way artists see their role as artists in the very difficult period that our societies are going through. The interest of these conversations between a sociologist of migration and a sample of urban artists whose personal history is directly marked by migration is based on the idea that they are key players in the post-migration and intercultural urban dynamics.

\section{Belgas artistas urbanos, la "crisis de la migración" y el terrorismo. Entrevistas con Kaer, Gioia Kayaga, Giacomo Lariccia, David Mendez Yepez, Tutu Puoane y Rival}

Basado en entrevistas con seis artistas de la escena musical urbana de las tres principales ciudades belgas (Amberes, Bruselas y Lieja), el artículo aborda tres cuestiones: la "crisis de la migración» en la actualidad, el impacto de los ataques terroristas que sacudieron al país y las políticas implementadas para responder y, por último, la forma en la cual los artistas ven su papel como artistas en el periodo tan difícil que atraviesan nuestras sociedades. El interés de estas conversaciones entre un sociólogo de la migración y un grupo de artistas urbanos cuya historia personal está directamente marcada por la migración se basa en la idea de que son actores clave en las dinámicas posteriores a la migración y en las dinámicas urbanas e interculturales. 